\title{
Juvenile Selenium Deficiency Impairs Cognition, Sensorimotor Gating, and Energy Homeostasis in Mice
}

\author{
Victor W. Kilonzo ${ }^{1}$, Alexandru R. Sasuclark ${ }^{1}$, Daniel J. Torres ${ }^{2}$, Celine Coyle ${ }^{1}$, \\ Jennifer M. Pilat ${ }^{3}$, Christopher S. Williams ${ }^{3}$ and Matthew W. Pitts ${ }^{1 *}$ \\ ${ }^{1}$ Department of Cell and Molecular Biology, University of Hawaii, Honolulu, HI, United States, ${ }^{2}$ Pacific Biosciences Research \\ Center, University of Hawaii at Manoa, School of Ocean and Earth Science and Technology (SOEST), Honolulu, HI, \\ United States, ${ }^{3}$ Department of Medicine and Cancer Biology, Vanderbilt University School of Medicine, Nashville, TN, \\ United States
}

OPEN ACCESS

Edited by:

Jong-Min Kim,

Seoul National University Bundang

Hospital, South Korea

Reviewed by:

Anna Kipp,

Friedrich Schiller University

Jena, Germany

Tommaso Filippini,

University of Modena and Reggio

Emilia, Italy

*Correspondence:

Matthew W. Pitts

mwpitts@hawaii.edu

Specialty section

This article was submitted to

Neuroenergetics, Nutrition and Brain

Health,

a section of the journal

Frontiers in Nutrition

Received: 13 February 2021 Accepted: 09 April 2021

Published: 07 May 2021

Citation:

Kilonzo VW, Sasuclark AR, Torres DJ,

Coyle C, Pilat JM, Williams CS and

Pitts MW (2021) Juvenile Selenium

Deficiency Impairs Cognition,

Sensorimotor Gating, and Energy

Homeostasis in Mice.

Front. Nutr. 8:667587.

doi: 10.3389/fnut.2021.667587
Selenium (Se) is an essential micronutrient of critical importance to mammalian life. Its biological effects are primarily mediated via co-translational incorporation into selenoproteins, as the unique amino acid, selenocysteine. These proteins play fundamental roles in redox signaling and includes the glutathione peroxidases and thioredoxin reductases. Environmental distribution of Se varies considerably worldwide, with concomitant effects on Se status in humans and animals. Dietary Se intake within a narrow range optimizes the activity of Se-dependent antioxidant enzymes, whereas both Se-deficiency and Se-excess can adversely impact health. Se-deficiency affects a significant proportion of the world's population, with hypothyroidism, cardiomyopathy, reduced immunity, and impaired cognition being common symptoms. Although relatively less prevalent, Se-excess can also have detrimental consequences and has been implicated in promoting both metabolic and neurodegenerative disease in humans. Herein, we sought to comprehensively assess the developmental effects of both Se-deficiency and Se-excess on a battery of neurobehavioral and metabolic tests in mice. Se-deficiency elicited deficits in cognition, altered sensorimotor gating, and increased adiposity, while Se-excess was surprisingly beneficial.

Keywords: selenium, sensorimotor gating, cognition, energy metabolism, neurodevelopment

\section{INTRODUCTION}

Selenium (Se) is an essential trace element in mammals, of which both deficiency and excess can have detrimental effects on health (1). Se supplementation within a narrow range optimizes activity of Se-dependent antioxidant enzymes that incorporate Se co-translationally in the form of selenocysteine. Se also counteracts the toxicity of certain heavy metals, such as arsenic, lead, and mercury $(2,3)$. Deficient Se intake impairs thyroid hormone metabolism and reduces activity of the antioxidant enzymes, glutathione peroxidase and thioredoxin reductase (4). In contrast, Se can be detrimental at high doses, with documented neurotoxic effects (5).

Se-deficiency is estimated to occur in roughly $10 \%$ of the world's population and is observed predominantly in regions with low soil Se-content, such as Scandinavia, New Zealand, and Northeast China (6). Furthermore, future climate change is predicted to decrease soil Se content in agricultural regions and augment the prevalence of Se-deficiency worldwide (7). Common symptoms associated with Se-deficiency include hypothyroidism, cardiomyopathy, compromised 
immunity, fatigue, and cognitive deficits (8-10). Also, altered serum Se levels have been documented in both autism and schizophrenia $(11,12)$, and it is hypothesized that redox imbalance during neurodevelopment increases risk for these neuropsychiatric conditions $(13,14)$.

On the opposite end of the spectrum, Se-excess can lead to toxicity and increased oxidative stress. In rodents, acute Se overexposure elicits motor deficits, catalepsy-like behavior and increased levels of dopamine, with inorganic selenium compounds being significantly more toxic than organic counterparts $(15,16)$. Moreover, in humans, rare cases of chronic Se-excess have been associated with elevated incidences of amyotrophic lateral sclerosis (17-19). Additionally, elevated selenium intake has been linked to higher incidences of type 2 diabetes (20), as have heightened levels of the selenium transport protein, selenoprotein $\mathrm{P}(21)$.

Whereas, the influence of Se has been extensively studied in many contexts, the developmental in vivo effects of chronic Se-deficiency and Se-excess upon measures of neurobehavior and energy metabolism have not been comprehensively characterized. Thus, we performed an expansive assessment of various behavioral and metabolic indices in young adult mice receiving dietary supplementation at levels corresponding to Se-deficient, Se-supplemented, and Se-excess upon weaning.

\section{MATERIALS AND METHODS}

\section{Animals}

All experiments were conducted on male C57BL/6J mice purchased from Jackson labs at 3-4 weeks of age. Mice were maintained on a 12-h light/dark cycle and provided ad libitum food and water access. Procedures and experimental protocols were approved by the University of Hawaii's Institutional Animal Care and Use Committee. All efforts were made to minimize animal discomfort and number of animals used.

\section{Diet}

Upon arrival at the University of Hawaii Animal Facility, mice were allocated into three groups, representing conditions of Sedeficiency, Se-supplementation, and Se-excess. All mice were administered Se-deficient laboratory chow $(\sim 0.08 \mathrm{ppm}$ Se $)$ (Research Diets, D19101Y), for which casein is the main source of both protein and Se, and the predominant Se species are organic. The Se-supplemented and Se-excess groups received sodium selenite in the drinking water at doses of $10 \mu \mathrm{M}$ and $100 \mu \mathrm{M}$, respectively. Hundred $\mu \mathrm{M}$ sodium selenite corresponds to $\sim 8$ ppm elemental Se, a dosage reported to induce mortality in rats (22) and elicit clinical symptoms in humans (23).

\section{Experimental Design}

Mice were group-housed until 10 weeks of age and then singlehoused 3 days prior to onset of behavioral experiments. Spatial learning was assessed on the Barnes maze at 10-12 weeks of age, followed by metabolic phenotyping at 14-16 weeks. Motor coordination was periodically examined at 8,12 , and 16 weeks of age. Testing for acoustic startle/prepulse inhibition was performed last, at 17-18 weeks, as this procedure involves loud auditory stimuli and could potentially confound other behavioral procedures. At 20 weeks of age, mice were euthanized via $\mathrm{CO}_{2}$ asphyxiation for collection of fresh tissue or deeply anesthetized (1.2\% Avertin; $0.7 \mathrm{ml} /$ mouse) and perfused with $4 \%$ paraformaldehyde for immunohistology. Blood was also collected upon sacrifice, and in non-perfused mice, fat depots for gonadal and inguinal white adipose tissue were collected and weighed. Brains from non-perfused mice were split along the sagittal plane, with one hemisphere used for Se analysis and the other hemisphere allocated for biochemical assays.

\section{Barnes Maze Test}

Spatial learning was assessed using the Barnes maze (TSE Systems) as described previously (24). In brief, the maze consists of a white circular board containing 40 equally spaced holes, with one hole leading to an escape tunnel. Mice were trained to find the escape tunnel, which remained at a fixed location relative to spatial cues for the duration of training. Training consisted of two trials daily ( 3 min max per trial) for 10 days, with the starting location varying pseudorandomly among the four quadrants. If a mouse failed to find the escape tunnel within the $3 \mathrm{~min}$ trial period, it was placed in the escape tunnel by the researcher and allowed to stay there for $15 \mathrm{~s}$. For each training trial, the latency to locate the escape tunnel and the number of incorrect holes checked (errors) before locating the escape tunnel were recorded. For analysis purposes, data were grouped into trial blocks, which consisted of 4 trials, with each trial administered from a distinct quadrant.

\section{Rotarod Test}

Starting speed for the Rotarod was $4 \mathrm{rpm}$ and increased to $40 \mathrm{rpm}$ over a $5 \mathrm{~min}$ period. The latency to fall off the rod was measured for each trial and the best score for each mouse was used for statistical analysis.

\section{Acoustic Startle and Prepulse Inhibition}

Mice were placed in the startle chamber (Responder-X, Columbus Instruments, Columbus, $\mathrm{OH}$ ) and allowed a 5min acclimation period with the background noise $(70 \mathrm{~dB})$ continually present. Following acclimation, two blocks of trials were administered to assess the acoustic startle response and prepulse inhibition, respectively, as described previously (25).

\section{Glycemic Control Testing}

Glucose tolerance was assessed by administering a glucose injection of $1 \mathrm{mg} / \mathrm{g}$ of body weight to animals that were fasted overnight. Tail blood was collected at time points $0,30,60,120$, and $180 \mathrm{~min}$ after injection and glucose levels were determined using strips and a glucometer (OneTouch Ultra, Lifescan).

\section{Lipid Droplet Analysis of Brown Adipose Tissue (BAT)}

BAT was collected from perfused animals ( $n=4$ per group), embedded in paraffin, sectioned at $5 \mu \mathrm{m}$, and stained with hematoxylin and eosin. Bright field images were taken at $20 \times$ magnification and imported into FIJI for image analysis. Images were thresholded, and droplets were measured using the 
"Analyze Particles" feature of FIJI. For each subject, 500-700 lipid droplets were measured.

\section{Metabolic Chambers}

Locomotion, respiratory metabolism, and ingestive behavior were measured using the PanLab OxyletPro ${ }^{\mathrm{TM}}$ System (Harvard Apparatus, Barcelona, Spain) according to the manufacturer's instructions. Mice were placed in individual chambers, with fresh bedding, food, and water, and allowed to acclimate for $24 \mathrm{~h}$, followed by $48 \mathrm{~h}$ of data collection. Cage air was sampled for $7 \mathrm{~min}$ periods every $35 \mathrm{~min}$ to measure oxygen and carbon dioxide concentrations. Data were collected and analyzed with Panlab METABOLISM software (Vídenská, Prague, Czech Republic).

\section{Protein Extraction and Immunoblotting}

Frozen tissues were lysed by sonication in CelLytic MT buffer (Sigma-Aldrich) containing protease inhibitors (Calbiochem) and centrifuged at $14,000 \mathrm{~g}$ for $10 \mathrm{~min}$ at $4^{\circ} \mathrm{C}$. Supernatants were collected and the protein concentrations were measured using the Bradford assay. For western blotting, $40 \mu \mathrm{g}$ samples of total protein were separated on 4-20\% SDS-PAGE gradient gels (BioRad), transferred to Immobilon-FL polyvinylidene difluoride membranes (Millipore), and probed for $2 \mathrm{~h}$ at room temperature with specific antibodies. Membranes were then incubated in the dark with secondary antibodies coupled to infrared fluorophores (LI-COR Biosciences). Blots were imaged and analyzed using an Odyssey infrared imager (LI-COR Biosciences). Relative protein levels were determined by dividing the optical density of the band representing the protein of interest by that of the loading control ( $\beta$-actin).

\section{Antibodies}

Primary antibodies used for Western blotting were as follows: goat anti-GPX1 (1:500; R\&D Systems, AF3798), mouse antiSELENBP1 (1:1,000, MBL, M061-3), rabbit anti-TXNRD2 $(1: 1,000$; Invitrogen, LF-PA0024), and rabbit anti- $\beta$-actin (1:5,000; Cell Signaling, 4970S).

\section{Leptin ELISA}

Serum leptin levels were measured using a commercially available solid-phase sandwich ELISA kit (Invitrogen) according to the manufacturer's instructions.

\section{Se Analysis}

Se was measured using a modification of the fluorometric assay of Koh and Benson (26) and Sheehan and Gao (27). Tissue was predigested in $6 \mathrm{ml}$ nitric acid at $150-300^{\circ} \mathrm{C}$ for $2 \mathrm{~h}$. Hundred $\mu \mathrm{l}$ predigested tissue, serum, or Se standard (Millipore Sigma, 89598) was then digested with $0.5 \mathrm{ml}$ perchloric:nitric acid $(1: 4)$ at $197^{\circ}$ for $1.5 \mathrm{~h}$. As samples cooled to $150^{\circ} \mathrm{C}$, $0.5 \mathrm{ml}$ hydrochloric acid $(\mathrm{HCl})$ was added and samples were maintained at $130-150^{\circ} \mathrm{C}$ for $30 \mathrm{~min}$. Next, $2 \mathrm{ml} 0.1 \mathrm{M}$ EDTA, $0.5 \mathrm{ml} \mathrm{2,3}$ diaminonaphthalene $(0.1 \% \mathrm{w} / \mathrm{v}$ in $0.1 \mathrm{M} \mathrm{HCl})$, and $3 \mathrm{ml}$ cyclohexane were added, followed by incubation at $60^{\circ} \mathrm{C}$ for $30 \mathrm{~min}$. Fluorescence was measured in a Perkin-Elmer LS 55 fluorometer and concentrations determined via comparison to a standard curve.

\section{Glutathione Peroxidase Activity Assay}

Soluble proteins were extracted as described above and normalized to a concentration of $4 \mathrm{mg} / \mathrm{ml}$. Glutathione peroxidase activity was measured as the reduction rate of cumene hydroperoxide catalyzed by the samples upon oxidation of nicotinamide adenine dinucleotide phosphate (NADPH) using a commercially available kit (Cayman Chemical). A unit of activity was defined as the consumption of $1 \mu \mathrm{mol}$ of NADPH per min, calculated from the expression $\left(V_{\max } \mathrm{X} V_{t /} V_{s}\right) /(0.0062 \mathrm{X}$ D), using $0.0062 \mu \mathrm{M}^{-1} \mathrm{~cm}^{-1}$ as the extinction coefficient for $\mathrm{NADPH}$ at $340 \mathrm{~nm}$.

\section{Statistical Analysis}

Data were analyzed and plotted using Prism software (GraphPad). Statistical tests varied according to the experiment and are indicated in the text and/or figure legends. To compare multiple groups, ANOVAs and Tukey's post-hoc test were utilized. All results are represented as mean \pm standard error of the mean (SEM).

\section{RESULTS}

For this study, newly weaned male mice were allocated into three groups devised to represent conditions of Se-deficiency (Se-def), Se-supplementation (Se-sup), and Se-excess (Se-exc). All mice were fed Se-deficient laboratory chow $(\sim 0.08 \mathrm{ppm}$ $\mathrm{Se}$ ), with the Se-sup and Se-exc groups receiving additional Se supplementation in their drinking water at doses of $10 \mu \mathrm{M}$ and $100 \mu \mathrm{M}$, respectively. As anticipated, we observed no differences among groups for food intake (Figure 1A), although water consumption did vary (Figure 1B) $\left[F_{(2,17)}=4.918, p=0.0206\right]$, with significant differences between the Se-def and Se-exc groups $(p=0.018)$. We also calculated Se intake based on water and food consumption, and mean values corresponded to $0.26,2.14$, and $13.94 \mu \mathrm{g} /$ days for the Se-def, Se-sup, and Se-exc groups, respectively (Figure 1C).

Upon sacrifice, tissue was harvested for determination of Se content and additional molecular analyses. For kidney $\left[F_{(2,9)}\right.$ $=78.91, p<0.0001]$, liver $\left[F_{(2,9)}=100.5, p<0.0001\right]$, and serum samples $\left[F_{(2,9)}=8.578, p=0.0082\right]$, Se levels differed among groups in a dose-dependent manner, whereas in brain $\left[F_{(2,8)}=0.7685, p=0.4951\right]$ and testes $\left[F_{(2,9)}=3.430, p=\right.$ 0.0781 , levels were comparable (Figure 1D). Parallel analyses of glutathione peroxidase (GPx) activity were conducted on liver, serum, and brain samples. Surprisingly, liver GPx activity $\left[F_{(2,9)}\right.$ $=2.256, p=0.1606]$ was similar between groups, whereas serum GPx activity $\left[F_{(2,9)}=0.5429, p=0.5937\right]$ showed similar nonsignificant trends as observed for Se analysis (Figure 1E). For brain, we detected a significant main effect of Se group upon GPx activity $\left[F_{(2,9)}=4.263, p=0.0498\right]$, with differences between the Se-def and Se-sup groups attaining significance $(p=0.0441)$. Western blotting was also performed on liver and brain samples to assess various markers of Se status. We probed for GPX1 and TXNRD2, two abundant selenoproteins known to be responsive and non-responsive to alterations in Se supply (28), respectively, and the selenium binding protein (SELENBP1), a putative factor protecting against Se-toxicity (29). For liver samples, levels of 


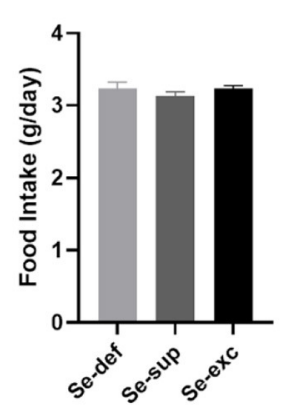

D

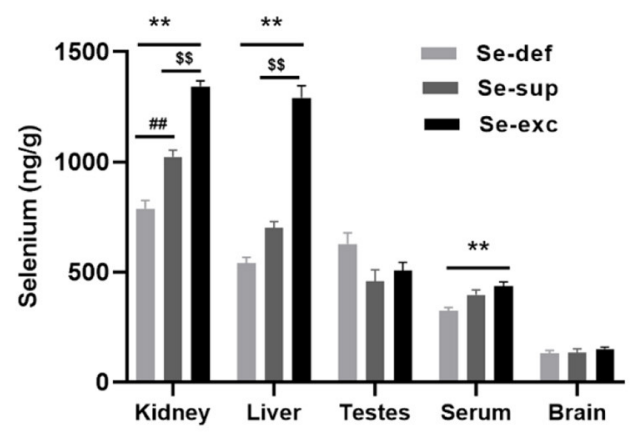

$\mathbf{F}$

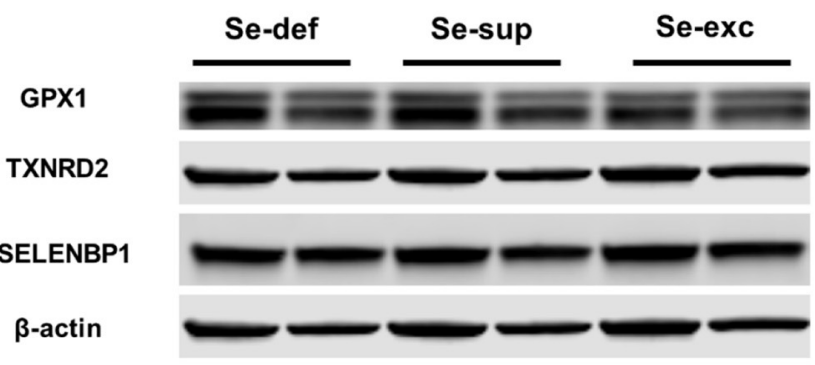

Brain Samples

H

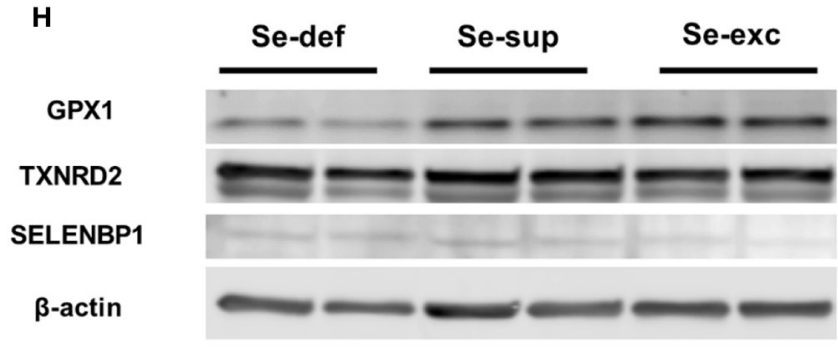

B

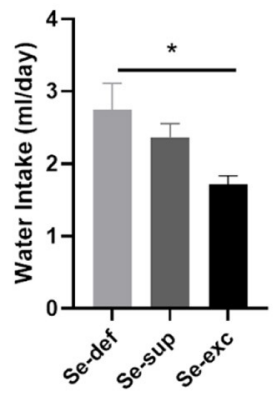

C

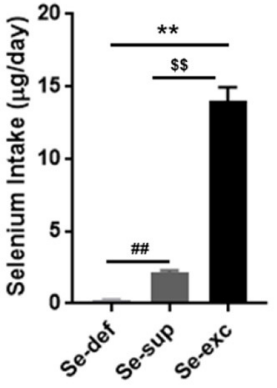

E

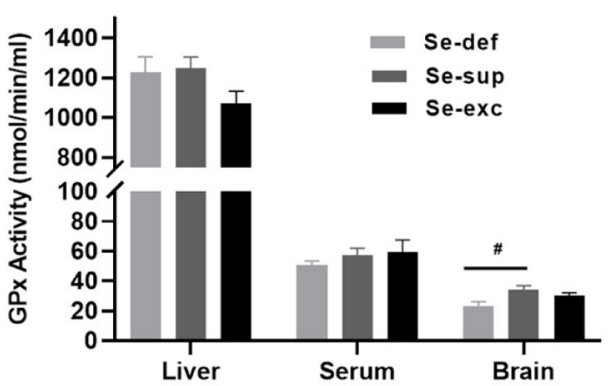

G

Liver Samples

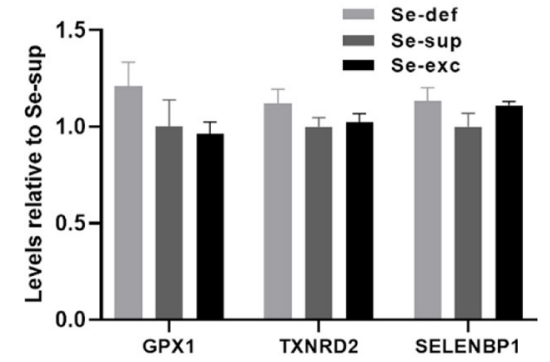

\section{Brain Samples}

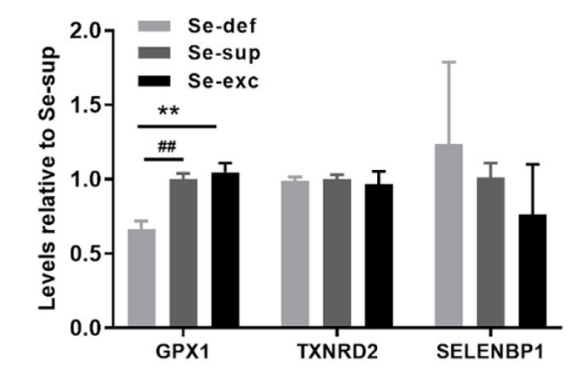

FIGURE 1 | Assessment of varying Se supplementation on organ-specific Se content, glutathione peroxidase activity, and selenoprotein levels. (A) Mean ( \pm SEM) daily food intake. (B) Mean ( \pm SEM) daily water consumption. (C) Mean ( \pm SEM) daily selenium intake ( $n=6-7$ per group). (D) Mean ( \pm SEM) selenium content in kidney, liver, testes, serum, and brain ( $n=3-4$ per group). (E) Mean ( \pm SEM) GPX activity in liver, serum, and brain $(n=4-6)$. (F-I) Protein levels of GPX1, TXNRD2, and SELENBP1 in liver $(\mathbf{F}, \mathbf{G})$ and brain $(\mathbf{H}, \mathbf{I})(n=4) .{ }^{\$ \$} p<0.01$ between Se-exc and Se-sup groups; ${ }^{*} p<0.05$ between Se-exc and Se-def groups; ${ }^{*} p<0.01$ between Se-exc and Se-def groups; ${ }^{*} p<0.05$ between Se-def and Se-sup groups; $\# \# p<0.01$ between Se-def and Se-sup groups.

GPX1, TXNRD2, and SELENBP1 were not impacted by Se group (Figures 1F,G). Brain levels of TXNRD2 and SELENBP1 were comparable between groups, but we did observe altered levels of
GPX1 $\left[F_{(2,9)}=15.67, p=0.0012\right]$, as levels were significantly reduced in the Se-def group (vs Se-sup: $p=0.0038$; vs. Se-exc: $p=0.0016)($ Figures 1H,I). 
Prior to harvesting of tissue, mice were subjected to a battery of neurobehavioral and metabolic tests. Cognition was evaluated using the Barnes maze, a widely utilized paradigm for spatial learning in rodents. Mice were trained to find a hidden escape tunnel located beneath one of 40 holes on the periphery of the circular maze. As anticipated, we observed a main effect of time on spatial learning, as indicated by less primary errors $\left[F_{(5,100)}=\right.$ $88.6, p<0.001]$ and a faster primary latency $\left(F_{(5,100)}=66.7, p<\right.$ 0.0001 ] when locating the escape tunnel (Figures 2A,B). We also detected a main effect of Se group upon the number of primary errors $\left[F_{(2,25)}=4.907, p=0.0159\right]$, but not upon the primary latency $\left[F_{(2,25)}=1.44, p=0.2560\right]$. For both primary errors $\left[F_{(8,100)}=1.697, p=0.1083\right]$ and primary latency $\left[F_{(8,100)}=\right.$ $1.015, p=0.4295]$, the time $\times$ Se group interaction was nonsignificant. Post-hoc analyses revealed higher levels of primary errors in the Se-def group during trial block one (vs. Se-exc: $p$ $=0.0151$ ) and two (vs Se-sup: $p=0.0008$; vs. Se-exc: $p=0.0233$ ). Likewise, primary latencies were significantly higher in the Se-def group during trial block 2 (vs Se-sup: $p=0.0146$; vs. Se-exc: $p=$ 0.0144 ). No significant differences were observed between groups for these measures during the remaining trial blocks nor during a probe trial conducted after trial block 5 (data not shown). As a whole, these results indicate that spatial learning is impaired by Se-deficiency.

Motor coordination, as determined by the latency to fall off a rotating rod of increasing speed, was examined at 8,12 , and 16 weeks of age (Figure 2C). Two-way ANOVA analyses revealed a main effect of time $\left[F_{(2,50)}=7.826, p=0.0011\right)$ and a significant time $\mathrm{x}$ Se group interaction effect $\left[F_{(4,50)}=2.779, p=0.0367\right]$, whereas the influence of Se group was non-significant $\left[F_{(2,25)}\right.$ $=1.208, p=0.3156]$. In our initial test at 8 weeks of age, we observed differences between the Se-sup and Se-exc groups, with Se-exc mice performing significantly worse $(p=0.0252)$. Surprisingly, motor coordination improved over time in the Seexc group, whereas performance declined in both the Se-def and Se-sup groups.

To assess sensorimotor gating, mice were tested for acoustic startle reactivity and prepulse inhibition. For acoustic startle (Figure 2D), we observed a main effect for stimulus intensity $\left[F_{(6,150)}=53.31, p<0.0001\right)$, whereas both Se group $\left[F_{(2,25)}\right.$ $=2.241, p=0.1273]$ and the stimulus intensity $\times$ Se group interaction $\left[F_{(12,150)}=1.610, p=0.0942\right]$ were not significant. Across the vast majority of stimulus intensities, startle magnitude was most pronounced in the Se-exc group, with statistically significant differences detected at $95 \mathrm{~dB}$ (vs. Se-sup: $p=0.0410$ ) and $110 \mathrm{~dB}$ (vs. Se-def: $p=0.0025$ ). In testing for prepulse inhibition (Figure 2E), a main effect of prepulse intensity $\left[F_{(2,50)}\right.$ $=16.07, p<0.0001]$ was found, while the effects of Se group $\left[F_{(2,25)}=1.062, p=0.3608\right]$ and the prepulse intensity $\times$ Se group interaction $\left[F_{(4,50)}=2.185, p=0.0841\right]$ failed to reach significance. Moreover, at the highest prepulse intensity (16 $\mathrm{dB})$, the Se-def group exhibited significantly reduced inhibition relative to the Se-exc group $(p=0.0361)$.

Mice were also tested for glycemic control and body weight was regularly monitored. For glucose tolerance testing (Figure 3A), a main effect of time $\left[F_{(4,76)}=63.18, p<0.0001\right]$ and a significant time $\mathrm{x}$ Se group interaction effect $\left[F_{(8,76)}\right.$
$=2.454, p=0.0203]$ was detected, whereas the effect of Se group was non-significant $\left[F_{(2,19)}=1.680, p=0.2129\right]$. Post-hoc tests revealed significantly elevated blood glucose levels $120 \mathrm{~min}$ after glucose injection in the Se-def group relative to the Seexc group $(p=0.0148)$. With respect to body weight, twoway ANOVA analysis revealed a main effect of time $\left[F_{(8,200)}=\right.$ 316.1, $p<0.0001$ ], with non-significant effects observed for Se group $\left[F_{(2,25)}=2.307, p=0.1203\right]$ and the time $\times$ Se group interaction $\left[F_{(16,200)}=0.4277, p=0.9740\right]$. Levels gradually diverged over time between the Se-def and Se-exc groups, with differences reaching significance at $20 \mathrm{wks}$ (Figure 3B) $(p=$ 0.0443). Upon sacrifice, we also found that relative levels of inguinal white adipose tissue (iWAT) differed between groups (Figure 3C) $\left[F_{(2,9)}=6.227, p=0.0201\right]$, with statistically significant differences between the Se-def and Se-exc groups ( $p$ $=0.0165)$. Similar non-significant trends were also observed for gonadal white adipose tissue (gWAT) (Figure 3D) $\left[F_{(2,9)}=\right.$ $2.119, p=0.1762]$ and serum leptin (Figure $3 E$ ) $\left[F_{(2,21)}=2.563\right.$, $p=0.1009]$. Finally, average lipid droplet size in brown adipose tissue (BAT) significantly differed between groups (Figures 3F,G) $\left[F_{(2,7,750)}=77.77, p<0.0001\right]$, as droplets were larger in the Se-def group $(p<0.0001)$.

At 14-16 weeks of age, mice were placed in metabolic chambers for 48-hrs to evaluate activity, respiratory metabolism, and ingestive behavior. For locomotion (Figures 4A,B), twoway ANOVA analysis detected main effects for both light cycle $\left[F_{(1,17)}=46.81, p<0.0001\right]$ and Se-group $\left[F_{(2,17)}=7.126\right.$, $p=0.0057]$, in conjunction with a non-significant light cycle $\mathrm{x}$ Se group interaction effect $\left[F_{(2,17)}=0.809, p=0.4617\right]$. During both the light $(p=0.0164)$ and dark cycles $(p=0.0018)$, the Se-exc group exhibited elevated locomotion relative to the Se-sup group. For measures of energy expenditure (Figure 4C) [EE: $\left.F_{(1,17)}=1,238, p<0.0001\right]$ and the respiratory quotient (Figure 4D) [RQ: $F_{(1,17)}=140.2, p<0.0001$ ], we also observed a main effect for the light cycle, but not for Se group [EE: $F_{(2,17)}=0.5002, p=0.6151$; RQ: $\left.F_{(2,17)}=3.126, p=0.0698\right]$. Moreover, we also detected a significant light cycle $\mathrm{x}$ Se group interaction effect for energy expenditure $\left[\mathrm{EE}: F_{(2,17)}=4.133, p\right.$ $=0.0344]$, but not for the respiratory quotient [RQ: $F_{(2,17)}=$ $1.212, p=0.3222]$.

\section{DISCUSSION}

In summary, these results detail the negative consequences of juvenile Se-deficiency upon measures of behavior and metabolism in early adulthood. Se-deficient mice displayed delayed learning and altered sensorimotor gating, and these deficits coincided with reduced GPx activity in brain. Moreover, Se-deficiency also resulted in impaired glycemic control, elevated body weight, and increased adiposity. Finally, Se-excess, at levels known to be toxic to humans, was surprisingly well-tolerated in mice and exerted beneficial effects on energy metabolism.

Our study corroborates prior findings that Se-deficiency hinders spatial learning $(30,31)$ and, to the best of our knowledge, represents the first association of Se-deficiency with impairments in sensorimotor gating. Deficits in cognition and sensorimotor 


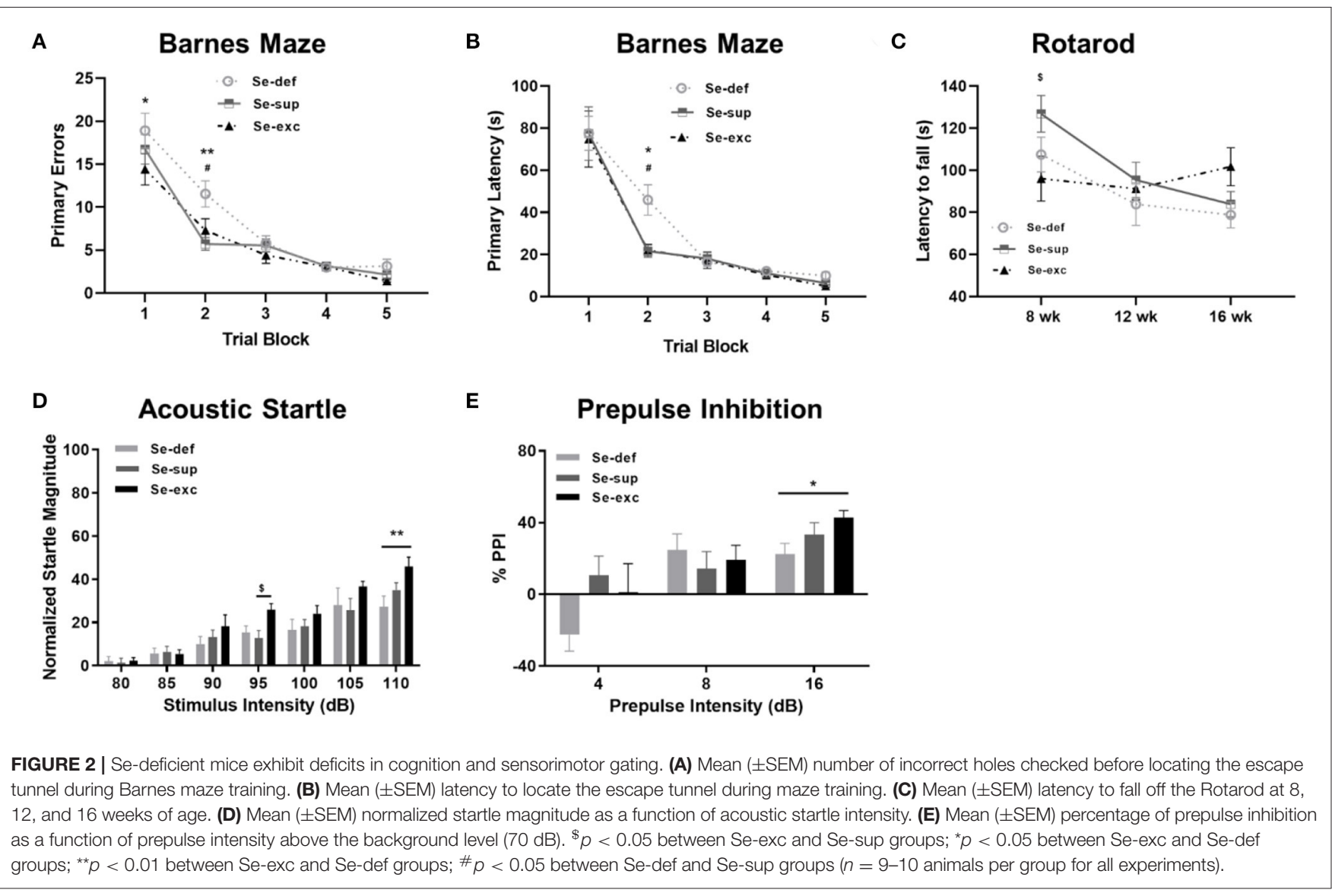

gating are hallmarks of many neurodevelopmental disorders, including schizophrenia and autism. For both schizophrenia $(11,32,33)$ and autism $(12,34,35)$, reduced Se levels have been chronicled in the literature, albeit there are many exceptions (36-38), and it is unclear whether this represents a cause or consequence of these conditions. Of particular significance to our results is a recent report examining the Se status of 287 Polish children, which were divided into four groups, corresponding to: (1) autism spectrum disorder (ASD) with obesity, (2) ASD without obesity, (3) non-ASD with obesity, and (4) non-ASD without obesity (12). Observed Se levels were lowest in ASD patients with obesity and highest in non-ASD patients without obesity, with differences between groups being highly significant $(p<0.001)$ for serum, urine, toenail samples. Moreover, across groups, Se levels were inversely correlated with body mass index $(\mathrm{p}<0.001)$ for all sample types.

The influence of Se supplementation upon energy metabolism is hotly debated and nuanced in the existing literature. An unanticipated corollary of the Nutritional Prevention of Cancer (NPC) trial was the observation that Se-supplementation (200 $\mu \mathrm{g}$ daily as Se-yeast) increased risk of type 2 diabetes for participants with baseline plasma Se levels within the upper tertile (20). Since these findings were documented, excess Se supplementation has been shown to adversely impact insulin signaling in multiple rodent models $(39,40)$. In contrast, reduced serum Se levels have been observed in morbidly obese patients (41), and supranutritional Se supplementation (240 $\mu \mathrm{g} /$ day) in the form of selenomethionine (SeMet) was recently shown to decrease both fat mass and circulating leptin levels in a 3-months dietary intervention study of obese individuals (42). Of potential relevance, we previously reported increased adiposity and elevated leptin levels in mice lacking SELENOM (43), an ER-resident selenoprotein that is highly expressed in brain and regulated by Se levels. Further studies showed that leptin upregulates SELENOM in hypothalamic neurons and that SELENOM, in turn, promotes leptin signaling (44). More recently, supranutrional Se supplementation $(2.25 \mathrm{ppm}$ SeMet in chow) was found to facilitate selenocysteine incorporation at sites canonically encoding cysteine, promote thermogenesis, and protect against diet-induced obesity (45).

One unexpected outcome of this study was the beneficial influence of $\mathrm{Se}$ at a dosage (8 ppm in water) originally hypothesized to elicit toxic effects. We chose to use chow that was mildly Se-deficient and provide further Se supplementation in the drinking water as selenite to the Se-sup and Se-exc groups. Inorganic Se species (selenite, selenate) are less readily absorbed by the intestine than organic counterparts (SeMet) (46-48), and are also significantly more toxic. For instance, the toxicity of selenite was found to be 53 -fold greater than that of SeMet when administered intracerebroventricularly to rats (15). With specific regard to supplementation of inorganic Se species in drinking water, increased mortality was previously reported at 


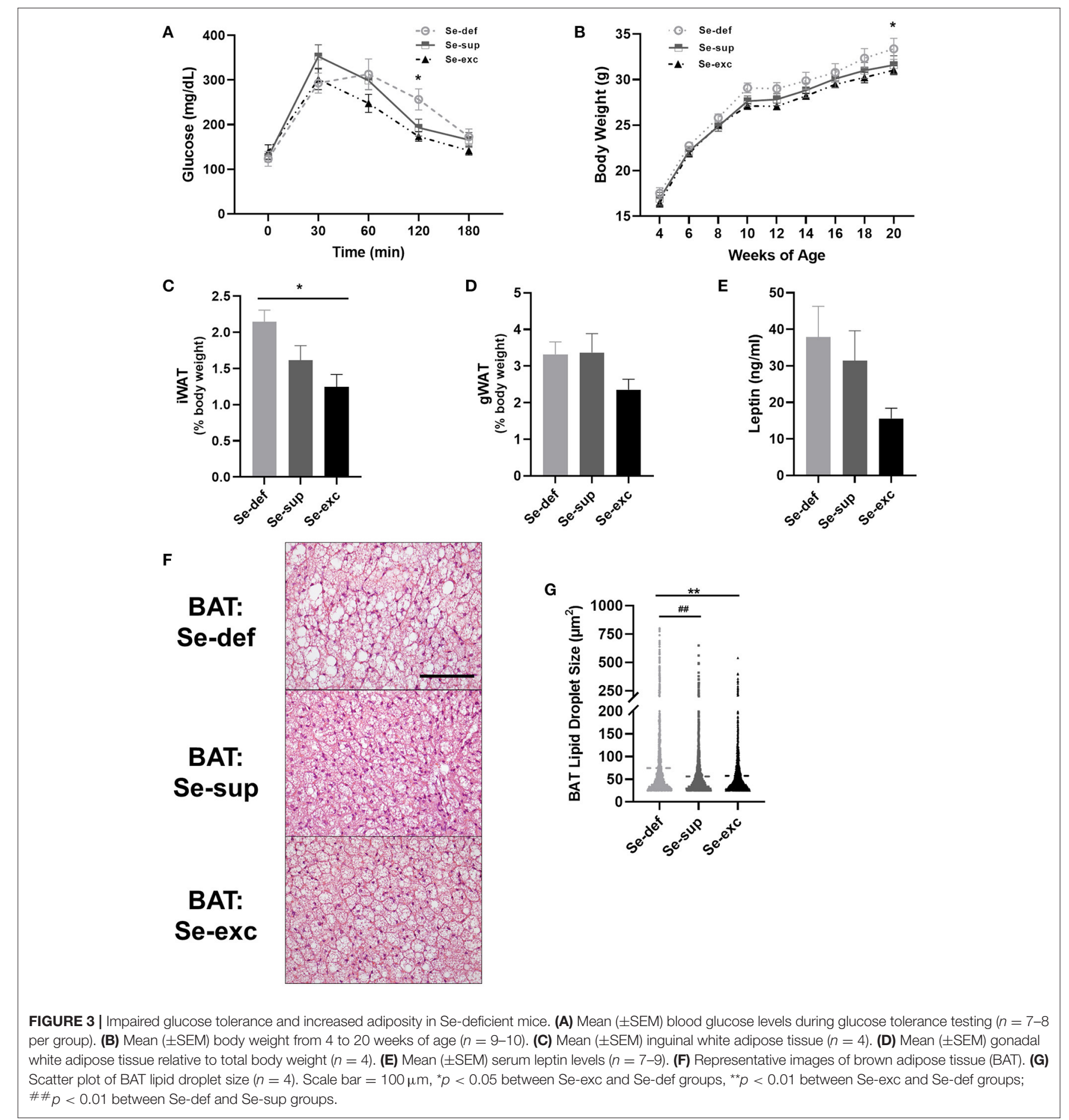

levels $>6$ ppm, although lower Se dosages (2-3 ppm) did lead to decreased body weights (22). Similarly, chow containing Se at $>5 \mathrm{ppm}$, has been shown to adversely impact growth and mortality in rodents (49) and pigs (50), with effects being more severe when selenite was the predominant Se species. Although relatively rare in humans, Se intoxication leads to loss of hair and nails, skin lesions, and nervous system abnormalities. A case study of Se toxicity in the Enshi district of China reported neurological defects in 18 of 22 subjects displaying signs of selenosis, and symptoms included hyperreflexia, convulsions, motor weakness, and hemiplegia (51). Moreover, blood Se levels in affected patients were observed to be roughly 100 times greater than subjects receiving a Se-adequate diet. Chronic Se overexposure has also been associated with an elevated risk 

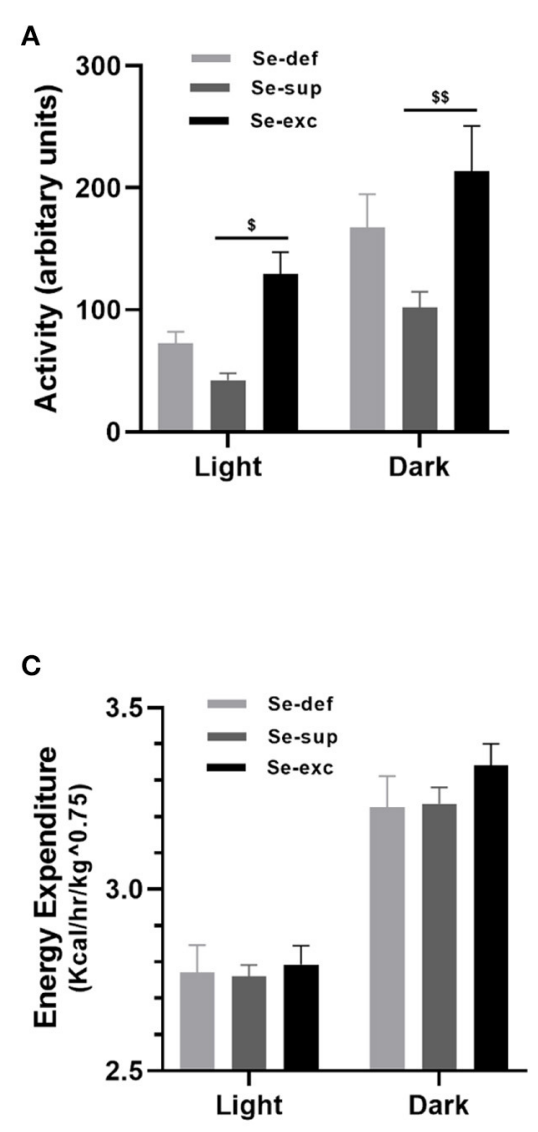

B

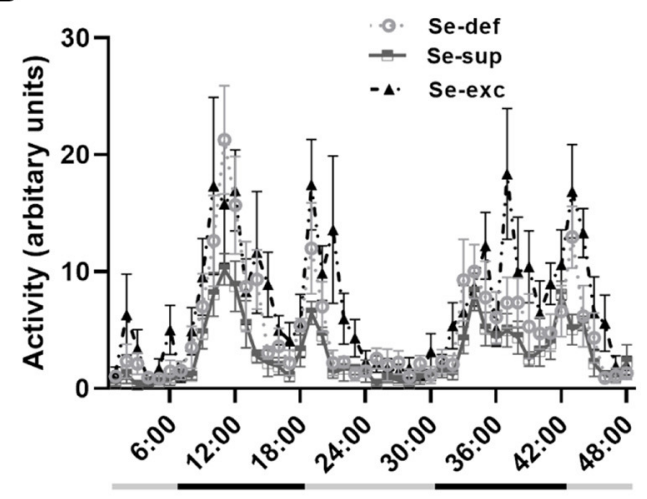

D

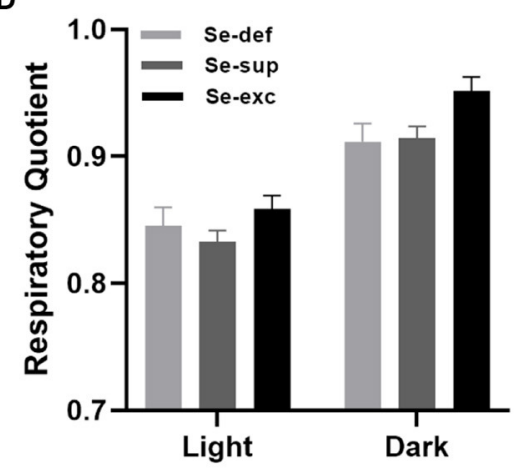

FIGURE 4 | Influence of varying Se supplementation on locomotion and respiratory metabolism. (A) Mean ( \pm SEM) locomotor activity during the light and dark cycles. (B) 48-h time course of locomotor activity. (C) Mean ( \pm SEM) energy expenditure during the light and dark cycles. (D) Mean ( \pm SEM) respiratory quotient during the light and dark cycles. ${ }^{\$} p<0.05$ between Se-exc and Se-sup groups; ${ }^{\$} p<0.01$ between Se-exc and Se-sup groups; ( $n=6-7$ animals per group for all experiments).

of neurodegenerative disease, specifically amyotrophic lateral sclerosis (ALS). This linkage was first noted in 1977, when a cluster of ALS cases was reported in a seleniferous area of South Dakota (19) and further substantiated by increased incidences of ALS in an Italian population chronically exposed (19741988) to drinking water containing high levels of selenate (18). Of further significance, elevated levels of selenite have been reported in the cerebrospinal fluid (CSF) of newly diagnosed ALS patients (52).

Another unanticipated finding was that Se supplementation modulated GPx activity to a greater extent in brain than liver. Brain Se levels are typically lower than other organs and blood (53), with Se homeostasis in the nervous system being tightly regulated by the blood-brain barrier (BBB) (54). Se transport to brain is regulated by endothelial cell-mediated uptake of SELENOP via the lipoprotein-related receptor, ApoER2, at the $\operatorname{BBB}(54,55)$. SELENOP is also expressed in astrocytes (56-58), especially those lining the $\mathrm{BBB}$, and astrocyte-derived SELENOP is speculated to supply ApoER2-expressing neurons with Se within the parenchyma (59). In cases of severe Se-deficiency, it is known that the brain and testes preferentially retain Se at the expense of other organs, and this phenomenon is dependent upon SELENOP and ApoER2 (55, 60-62). It should be duly noted that our Se-deficient chow contained 0.08 ppm Se, severalfold higher than that of many Se deprivation studies, but still well below the $0.15 \mathrm{ppm}$ minimum recommended for rodent diets by the AIN (63). Given that liver and kidney represent the primary sites of Se metabolism and excretion (64), respectively, the fact that supplementation most impacted Se content in these tissues was expected. The effect of supplementation on serum Se was less robust, suggesting that most Se was converted to excretory metabolites in liver, with a small fraction being incorporated into SELENOP. Moreover, it appears that our Sedeficient diet did not affect liver GPx activity, in line with prior findings by Sunde and colleagues showing that hepatic levels of GPx activity plateau when dietary Se is $0.09 \mathrm{ppm}$ or greater (65). Furthermore, prior evidence suggests that supplementation at levels similar to our study can significantly impact brain GPx activity. For example, Whanger and colleagues reported that increasing dietary Se content from 0.1 to $4 \mathrm{ppm}$ raised GPX activity by 32 and $77 \%$ in cortex and cerebellum, respectively (66).

It is imperative to note this study has several caveats that merit consideration. First, to reduce cost and animal usage, only 
male mice were used. Sex-specific differences in the biological effects of Se are well-documented in the literature (67-69), with males typically being more adversely impacted by deviations in Se intake. Second, experiments were conducted on young adult mice (3-5 months) and the possibility that long-term Se overexposure elicits neurodegenerative effects at later time points cannot be ruled out. Finally, given that our dietary intervention began shortly after weaning, it is probable that Se supplementation triggered developmental epigenetic adaptations to cope with Se-excess. Interestingly, Se-excess mice performed significantly worse in the initial rotarod test, but their performance improved over time, while that of the other two groups (Se-def, Se-sup) declined. It is quite possible that providing adult mice with Se at our chosen excessive dose may elicit detrimental toxic effects not observed in juveniles.

Nevertheless, these results detail the adverse effects of mild Se-deficiency and suggest that juvenile Se status is critical for optimal neurodevelopment. These findings may have important implications for future prevention and treatment of neurodevelopmental disorders where redox imbalance is a key characteristic.

\section{DATA AVAILABILITY STATEMENT}

The raw data supporting the conclusions of this article will be made available by the authors, without undue reservation.

\section{REFERENCES}

1. Koller LD, Exon JH. The two faces of selenium-deficiency and toxicity-are similar in animals and man. Can J Vet Res. (1986) 50:297-306.

2. Ikemoto T, Kunito T, Tanaka H, Baba N, Miyazaki N, Tanabe S. Detoxification mechanism of heavy metals in marine mammals and seabirds: interaction of selenium with mercury, silver, copper, zinc, and cadmium in liver. Arch Environ Contam Toxicol. (2004) 47:402-13. doi: 10.1007/s00244-004-3188-9

3. Berry MJ, Ralston NV. Mercury toxicity and the mitigating role of selenium. Ecohealth. (2008) 5:456-9. doi: 10.1007/s10393-008-0204-y

4. Rayman MP, The importance of selenium to human health. Lancet. (2000) 356:233-41. doi: 10.1016/S0140-6736(00)02490-9

5. Vinceti M, Mandrioli J, Borella P, Michalke B, Tsatsakis A, Finkelstein Y. Selenium neurotoxicity in humans: bridging laboratory and epidemiologic studies. Toxicol Lett. (2014) 230:295-303. doi: 10.1016/j.toxlet.2013.11.016

6. Combs GF Jr. Selenium in global food systems. Br J Nutr. (2001) 85:51747. doi: 10.1079/BJN2000280

7. Jones GD, Droz B, Greve P, Gottschalk P, Poffet D, McGrath SP, et al. Selenium deficiency risk predicted to increase under future climate change. Proc Natl Acad Sci USA. (2017) 114:2848-53. doi: 10.1073/pnas.1611576114

8. Gladyshev VN, Arner ES, Berry MJ, Brigelius-Flohe R, Bruford EA, Burk RF, et al. Selenoprotein Gene Nomenclature. J Biol Chem. (2016) 291:2403640. doi: 10.1074/jbc.M116.756155

9. Hoffmann PR, Berry MJ. The influence of selenium on immune responses. Mol Nutr Food Res. (2008) 52:1273-80. doi: 10.1002/mnfr.200700330

10. Kohrle J, Jakob F, Contempre B, Dumont JE. Selenium, the thyroid, and the endocrine system. Endocr Rev. (2005) 26:944-84. doi: 10.1210/er.2001-0034

11. Cai L, Chen T, Yang J, Zhou K, Yan X, Chen W, et al. Serum trace element differences between Schizophrenia patients and controls in the Han Chinese population. Sci Rep. (2015) 5:15013. doi: 10.1038/srep15013

12. Blazewicz A, Szymanska I, Dolliver W, Suchocki P, Turlo J, Makarewicz A, et al. Are obese patients with autism spectrum disorder more likely to be selenium deficient? Research findings on pre- and post-pubertal children. Nutrients. (2020) 12:3581. doi: 10.3390/nu12113581

\section{ETHICS STATEMENT}

The animal study was reviewed and approved by University of Hawaii's Institutional Animal Care and Use Committee.

\section{AUTHOR CONTRIBUTIONS}

VK and MP designed the experiments. VK, AS, DT, CC, JP, and MP performed research. CW contributed reagents/analytic tools. MP analyzed data and wrote the paper. All authors contributed to the article and approved the submitted version.

\section{FUNDING}

This research was supported by National Institutes of Health Grants U54 MD007601, P20 GM103466, F32DK124963-02, (to DT), and a Faculty Mentoring Grant (to MP) from the Undergraduate Research Opportunities Program, Office of the Vice Chancellor for Research at the University of Hawaii at Manoa.

\section{SUPPLEMENTARY MATERIAL}

The Supplementary Material for this article can be found online at: https://www.frontiersin.org/articles/10.3389/fnut.2021. 667587/full\#supplementary-material

13. Steullet P, Cabungcal JH, Coyle J, Didriksen M, Gill K, Grace AA, et al. Oxidative stress-driven parvalbumin interneuron impairment as a common mechanism in models of schizophrenia. Mol Psychiatry. (2017) 22:93643. doi: 10.1038/mp.2017.47

14. Bjorklund G, Meguid NA, El-Bana MA, Tinkov AA, Saad K, Dadar M, et al. Oxidative stress in autism spectrum disorder. Mol Neurobiol. (2020) 57:2314-32. doi: 10.1007/s12035-019-01742-2

15. Ammar EM, Couri D. Acute toxicity of sodium selenite and selenomethionine in mice after ICV or IV administration. Neurotoxicology. (1981) 2:383-6.

16. Rasekh HR, Davis MD, Cooke LW, Mazzio EA, Reams RR, Soliman KF. The effect of selenium on the central dopaminergic system: a microdialysis study. Life Sci. (1997) 61:1029-35. doi: 10.1016/S0024-3205(97)00610-3

17. Vinceti M, Bonvicini F, Rothman KJ, Vescovi L, Wang F. The relation between amyotrophic lateral sclerosis and inorganic selenium in drinking water: a population-based case-control study. Environ Health. (2010) 9:77. doi: 10.1186/1476-069X-9-77

18. Vinceti M, Guidetti D, Pinotti M, Rovesti S, Merlin M, Vescovi $\mathrm{L}$, et al. Amyotrophic lateral sclerosis after long-term exposure to drinking water with high selenium content. Epidemiology. (1996) 7:52932. doi: 10.1097/00001648-199609000-00014

19. Kilness AW, Hichberg FH. Amyotrophic lateral sclerosis in a high selenium environment. JAMA. (1977) 237:2843-4. doi: 10.1001/jama.237.26.2843

20. Stranges S, Marshall JR, Natarajan R, Donahue RP, Trevisan M, Combs $\mathrm{GF}$, et al. Effects of long-term selenium supplementation on the incidence of type 2 diabetes: a randomized trial. Ann Intern Med. (2007) 147:2173. doi: 10.7326/0003-4819-147-4-200708210-00175

21. Misu H, Takamura T, Takayama H, Hayashi H, Matsuzawa-Nagata N, Kurita $\mathrm{S}$, et al. A liver-derived secretory protein, selenoprotein $\mathrm{P}$, causes insulin resistance. Cell Metab. (2010) 12:483-95. doi: 10.1016/j.cmet.2010.09.015

22. Palmer IS, Olson OE. Relative toxicities of selenite and selenate in the drinking water of rats. J Nutr. (1974) 104:306-14. doi: 10.1093/jn/104.3.306

23. Rosenfeld I, Beath OA. Selenium: Geobotany, Biochemistry, Toxicity, and Nutrition. New York, NY: Academic Press (1964). doi: 10.1016/B978-1-4832-2800-6.50005-5 
24. Pitts MW. Barnes maze procedure for spatial learning and memory in mice. Bio Protoc. (2018) 8:2744. doi: 10.21769/BioProtoc.2744

25. Pitts MW, Raman AV, Hashimoto AC, Todorovic C, Nichols RA, Berry MJ. Deletion of selenoprotein $\mathrm{P}$ results in impaired function of parvalbumin interneurons and alterations in fear learning and sensorimotor gating. Neuroscience. (2012) 208:58-68. doi: 10.1016/j.neuroscience.2012.02.017

26. Koh TS, Benson TH. Critical re-appraisal of fluorometric method for determination of selenium in biological materials. J Assoc Off Anal Chem. (1983) 66:918-26. doi: 10.1093/jaoac/66.4.918

27. Sheehan TM, Gao M. Simplified fluorometric assay of total selenium in plasma and urine. Clin Chem. (1990) 36:2124-6. doi: 10.1093/clinchem/36.12.2124

28. Seyedali A, Berry MJ. Nonsense-mediated decay factors are involved in the regulation of selenoprotein mRNA levels during selenium deficiency. RNA. (2014) 20:1248-56. doi: 10.1261/rna.043463.113

29. Kohnlein K, Urban N, Guerrero-Gomez D, Steinbrenner H, Urbanek P, Priebs $\mathrm{J}$, et al. A Caenorhabditis elegans ortholog of human selenium-binding protein 1 is a pro-aging factor protecting against selenite toxicity. Redox Biol. (2020) 28:101323. doi: 10.1016/j.redox.2019.101323

30. Babur E, Tan B, Yousef M, Cinbas S, Suer C, Dursun N. Deficiency but not supplementation of selenium impairs the hippocampal long-term potentiation and hippocampus-dependent learning. Biol Trace Elem Res. (2019) 192:252-62. doi: 10.1007/s12011-019-01666-x

31. Watanabe C, Satoh H. Effects of prolonged selenium deficiency on open field behavior and Morris water maze performance in mice. Pharmacol Biochem Behav. (1995) 51:747-52. doi: 10.1016/0091-3057(95)00025-R

32. Liu T, Lu QB, Yan L, Guo J, Feng F, Qiu J, et al. Comparative study on serum levels of 10 trace elements in schizophrenia. PLoS ONE. (2015) 10:e0133622. doi: 10.1371/journal.pone.0133622

33. Ma J, Yan L, Guo T, Yang S, Liu Y, Xie Q, et al. Association between serum essential metal elements and the risk of schizophrenia in China. Sci Rep. (2020) 10:10875. doi: 10.1038/s41598-020-66496-7

34. Lakshmi Priya MD, Geetha A. Level of trace elements (copper, zinc, magnesium and selenium) and toxic elements (lead and mercury) in the hair and nail of children with autism. Biol Trace Elem Res. (2011) 142:14858. doi: 10.1007/s12011-010-8766-2

35. El-Ansary A, Bjorklund G, Tinkov AA, Skalny AV, Al Dera H. Relationship between selenium, lead, and mercury in red blood cells of Saudi autistic children. Metab Brain Dis. (2017) 32:1073-80. doi: 10.1007/s11011-017-9996-1

36. Yanik M, Kocyigit A, Tutkun H, Vural H, Herken H. Plasma manganese, selenium, zinc, copper, and iron concentrations in patients with schizophrenia. Biol Trace Elem Res. (2004) 98:109-17. doi: 10.1385/BTER:98:2:109

37. Skalny AV, Simashkova NV, Klyushnik TP, Grabeklis AR, Bjorklund G, Skalnaya MG, et al. Hair toxic and essential trace elements in children with autism spectrum disorder. Metab Brain Dis. (2017) 32:195202. doi: 10.1007/s11011-016-9899-6

38. Vidovic B, Dordevic B, Milovanovic S, Skrivanj S, Pavlovic Z, Stefanovic A, et al. Selenium, zinc, and copper plasma levels in patients with schizophrenia: relationship with metabolic risk factors. Biol Trace Elem Res. (2013) 156:228. doi: 10.1007/s12011-013-9842-1

39. Zeng MS, Li X, Liu Y, Zhao H, Zhou JC, Li K, et al. A high-selenium diet induces insulin resistance in gestating rats and their offspring. Free Radic Biol Med. (2012) 52:1335-42. doi: 10.1016/j.freeradbiomed.2012.01.017

40. Labunskyy VM, Lee BC, Handy DE, Loscalzo J, Hatfield DL, Gladyshev VN. Both maximal expression of selenoproteins and selenoprotein deficiency can promote development of type 2 diabetes-like phenotype in mice. Antioxid Redox Signal. (2011) 14:2327-36. doi: 10.1089/ars.20 10.3526

41. Alasfar F, Ben-Nakhi M, Khoursheed M, Kehinde EO, Alsaleh M. Selenium is significantly depleted among morbidly obese female patients seeking bariatric surgery. Obes Surg. (2011) 21:1710-3. doi: 10.1007/s11695-0110458-2

42. Cavedon E, Manso J, Negro I, Censi S, Serra R, Busetto L, et al. Selenium supplementation, body mass composition, and leptin levels in patients with obesity on a balanced mildly hypocaloric diet: a pilot study. Int J Endocrinol. (2020) 2020:4802739. doi: 10.1155/2020/4802739
43. Pitts MW, Reeves MA, Hashimoto AC, Ogawa A, Kremer P, Seale LA, et al. Deletion of selenoprotein M leads to obesity without cognitive deficits. J Biol Chem. (2013) 288:26121-34. doi: 10.1074/jbc.M113.471235

44. Gong T, Hashimoto AC, Sasuclark AR, Khadka VS, Gurary A, Pitts MW. Selenoprotein M promotes hypothalamic leptin signaling and thioredoxin antioxidant activity. Antioxid Redox Signal. (2019) doi: 10.1089/ars.2018.7594

45. Jedrychowski MP, Lu GZ, Szpyt J, Mariotti M, Garrity R, Paulo JA, et al. Facultative protein selenation regulates redox sensitivity, adipose tissue thermogenesis, and obesity. Proc Natl Acad Sci USA. (2020) 117:10789796. doi: $10.1073 /$ pnas. 2001387117

46. Daniels LA. Selenium metabolism and bioavailability. Biol Trace Elem Res. (1996) 54:185-99. doi: 10.1007/BF02784430

47. Vendeland SC, Deagen JT, Butler JA, Whanger PD. Uptake of selenite, selenomethionine and selenate by brush border membrane vesicles isolated from rat small intestine. Biometals. (1994) 7:305-12. doi: 10.1007/BF00144126

48. Whanger P, Vendeland S, Park YC, Xia Y. Metabolism of subtoxic levels of selenium in animals and humans. Ann Clin Lab Sci. (1996) 26:99-113.

49. Raines AM, Sunde RA. Selenium toxicity but not deficient or super-nutritional selenium status vastly alters the transcriptome in rodents. BMC Genomics. (2011) 12:26. doi: 10.1186/1471-2164-12-26

50. Kim YY, Mahan DC. Comparative effects of high dietary levels of organic and inorganic selenium on selenium toxicity of growing-finishing pigs. J Anim Sci. (2001) 79:942-8. doi: 10.2527/2001.794942x

51. Yang GQ, Wang SZ, Zhou RH, Sun SZ. Endemic selenium intoxication of humans in China. Am J Clin Nutr. (1983) 37:872-1. doi: 10.1093/ajen/37.5.872

52. Vinceti M, Solovyev N, Mandrioli J, Crespi CM, Bonvicini F, Arcolin E, et al. Cerebrospinal fluid of newly diagnosed amyotrophic lateral sclerosis patients exhibits abnormal levels of selenium species including elevated selenite. Neurotoxicology. (2013) 38:25-32. doi: 10.1016/j.neuro.2013.05.016

53. Burk RF, Hill KE. Selenoprotein P-expression, functions, and roles in mammals. Biochim Biophys Acta. (2009) 1790:14417. doi: 10.1016/j.bbagen.2009.03.026

54. Burk RF, Hill KE, Motley AK, Winfrey VP, Kurokawa S, Mitchell SL, et al. Selenoprotein $\mathrm{P}$ and apolipoprotein $\mathrm{E}$ receptor-2 interact at the bloodbrain barrier and also within the brain to maintain an essential selenium pool that protects against neurodegeneration. FASEB J. (2014) 28:357988. doi: 10.1096/fj.14-252874

55. Burk RF, Hill KE, Olson GE, Weeber EJ, Motley AK, Winfrey VP, et al. Deletion of apolipoprotein E receptor-2 in mice lowers brain selenium and causes severe neurological dysfunction and death when a low-selenium diet is fed. J Neurosci. (2007) 27:6207-11. doi: 10.1523/JNEUROSCI.1153-07.2007

56. Steinbrenner H, Alili L, Bilgic E, Sies H, Brenneisen P. Involvement of selenoprotein $\mathrm{P}$ in protection of human astrocytes from oxidative damage. Free Radic Biol Med. (2006) 40:151323. doi: 10.1016/j.freeradbiomed.2005.12.022

57. Sasuclark AR, Khadka VS, Pitts MW. Cell-type specific analysis of selenium-related genes in brain. Antioxidants (Basel). (2019) 8:120. doi: $10.3390 /$ antiox 8050120

58. Yang X, Hill KE, Maguire MJ, Burk RF. Synthesis and secretion of selenoprotein P by cultured rat astrocytes. Biochim Biophys Acta. (2000) 1474:390-6. doi: 10.1016/S0304-4165(00)00035-0

59. Steinbrenner $\mathrm{H}$, Sies $\mathrm{H}$. Selenium homeostasis and antioxidant selenoproteins in brain: implications for disorders in the central nervous system. Arch Biochem Biophys. (2013) 536:152-7. doi: 10.1016/j.abb.2013.02.021

60. Hill KE, Zhou J, McMahan WJ, Motley AK, Atkins JF, Gesteland RF, et al. Deletion of selenoprotein P alters distribution of selenium in the mouse. J Biol Chem. (2003) 278:13640-6. doi: 10.1074/jbc.M300755200

61. Nakayama A, Hill KE, Austin LM, Motley AK, Burk RF. All regions of mouse brain are dependent on selenoprotein P for maintenance of selenium. J Nutr. (2007) 137:690-3. doi: 10.1093/jn/137.3.690

62. Akahoshi N, Anan Y, Hashimoto Y, Tokoro N, Mizuno R, Hayashi $\mathrm{S}$, et al. Dietary selenium deficiency or selenomethionine excess drastically alters organ selenium contents without altering the expression of most selenoproteins in mice. J Nutr Biochem. (2019) 69:120-9. doi: 10.1016/j.jnutbio.2019.03.020

63. Reeves PG, Nielsen FH, Fahey GC Jr. AIN-93 purified diets for laboratory rodents: final report of the American Institute of Nutrition ad hoc writing 
committee on the reformulation of the AIN-76A rodent diet. J Nutr. (1993) 123:1939-51. doi: 10.1093/jn/123.11.1939

64. Burk RF, Hill KE. Regulation of Selenium Metabolism and Transport. Annu Rev Nutr. (2015) 35:109-34. doi: 10.1146/annurev-nutr-071714-034250

65. Sunde RA, Raines AM. Selenium regulation of the selenoprotein and nonselenoprotein transcriptomes in rodents. Adv Nutr. (2011) 2:13850. doi: 10.3945/an.110.000240

66. Sun Y, Butler JA, Whanger PD. Glutathione peroxidase activity and selenoprotein $\mathrm{W}$ levels in different brain regions of selenium-depleted rats(1). J Nutr Biochem. (2001) 12:88-94. doi: 10.1016/S0955-2863(00)00130-3

67. Pitts MW, Kremer PM, Hashimoto AC, Torres DJ, Byrns CN, Williams $\mathrm{CS}$, et al. Competition between the brain and testes under seleniumcompromised conditions: insight into sex differences in selenium metabolism and risk of neurodevelopmental disease. J Neurosci. (2015) 35:1532638. doi: 10.1523/JNEUROSCI.2724-15.2015

68. Byrns CN, Pitts MW, Gilman CA, Hashimoto AC, Berry MJ. Mice lacking selenoprotein $\mathrm{P}$ and selenocysteine lyase exhibit severe neurological dysfunction, neurodegeneration, audiogenic seizures. J Biol Chem. (2014) 289:9662-74. doi: 10.1074/jbc.M113.540682

69. Schomburg L, Schweizer U. Hierarchical regulation of selenoprotein expression and sex-specific effects of selenium. Biochim Biophys Acta. (2009) 1790:1453-62. doi: 10.1016/j.bbagen.2009.03.015

Conflict of Interest: The authors declare that the research was conducted in the absence of any commercial or financial relationships that could be construed as a potential conflict of interest.

Copyright (C) 2021 Kilonzo, Sasuclark, Torres, Coyle, Pilat, Williams and Pitts. This is an open-access article distributed under the terms of the Creative Commons Attribution License (CC BY). The use, distribution or reproduction in other forums is permitted, provided the original author(s) and the copyright owner(s) are credited and that the original publication in this journal is cited, in accordance with accepted academic practice. No use, distribution or reproduction is permitted which does not comply with these terms. 